\title{
This Month in AJP
}

\section{Dysfunctional Cholesterol Metabolism in Retina}

Cholesterol metabolism is not well understood in the retina, where CYP27A1 and CYP46A1 are the major cholesterolmetabolizing enzymes. Using Cyp27a1 ${ }^{-1-}$ Cyp $46 a 1^{-1-}$ mice, Saadane et al (Am J Pathol 2014, 184:2403-2419) studied the significance of cholesterol metabolism in retina. Despite normal serum cholesterol and glucose levels, Cyp27a1 ${ }^{-1-}$ Cyp46a1 ${ }^{-1-}$ mice had changes in the retinal vasculature, retina, and several nonocular organs, and they exhibited retinal macrophage activation and oxidative stress in the photoreceptor inner segments, mimicking other retinal diseases. The affected organs developed pathology that reflected tissue response to cholesterol overload and impaired oxysterol production. Retinal cholesterol metabolism appears to be important for maintenance of the normal retina, suggesting a novel model for pharmacological evaluation of molecules for treatment of diseases affecting retinal vasculature.

\section{INPP4B Polymorphisms Linked to MS}

Nerve conduction impairment commonly occurs in multiple sclerosis (MS), and measurement of evoked potentials are widely used for diagnosis and prognosis. Lemcke et al (Am J Pathol 2014, 184:2420-2429) used a classical genetic approach to identify novel genes controlling nerve conduction in mice. Quantitative trait mapping identified EAE31, a locus controlling latency of motor-evoked potentials and clinical onset of experimental autoimmune encephalomyelitis (EAE). Detailed fine mapping identified inositol polyphosphate-4-phosphatase, type II (Inpp 4b) as the quantitative trait gene for EAE31. Transgenic mice of different strains carrying specific polymorphisms under the control of a ubiquitous neuronal promoter validated Inpp $4 b$ involvement. Interestingly, an association of INPP4B polymorphisms is also conserved in humans and was associated with MS incidence in German and Spanish cohorts. INPP4B polymorphisms are candidate prognostic markers for MS.

\section{KDM5C Is Associated with Poor Prognosis in Prostate Cancer}

Studies have implicated histone lysine demethylases (KDMs) in prostate cancer; however, the specific role of KDM5C remains unexplored. Stein et al (Am J Pathol 2014, 184:2430-2437) systematically studied the expression pattern and the biological relevance of KDM5C in two independent radical prostatectomy cohorts. Positive nuclear KDM5C staining significantly associated with a reduced prostate-specific antigen relapse-free survival. Nuclear KDM5C expression was confirmed as an independent prognostic parameter. Strikingly, the prognostic value of nuclear KDM5C expression for progression-free survival was exclusively pronounced for Gleason group 7. KDM5C knockdown retarded prostate cancer cell growth in vitro and induced regulation of several proliferation-associated genes. KDM5C thus represents a potential novel drug target in prostate cancer.

\section{F0X01 Accelerates Skin Wound Healing}

The full involvement and mechanism of action of the forkhead box O (FOXO) family of transcription factors in tissue-repair processes remains largely unknown. Mori et al (Am J Pathol 2014, 184:2465-2479) studied the molecular functions of FOXO family gene members in skin wound healing and their potential application in a clinical setting. Foxo1 and Foxo3a were both up-regulated during murine skin wound healing. $\mathrm{Foxol}^{+/-}$mice exhibited accelerated skin wound healing, enhanced keratinocyte migration, reduced inflammatory response, and decreased scarring; no wound phenotype was observed in Foxo $3 a^{-/-}$mice. Acute knockdown of the Foxo1 protein at wound sites improved the quality of healing. Similarly, altered FOXO1 expression in human patients was associated with the development of keloids (growing scars), in a race-dependent manner. Molecular targeting of FOXO1 may improve the quality of healing and reduce pathological scarring.

\section{SDC4 Regulates MMP-3 Expression in Disc Degeneration}

The relationship between matrix metalloproteinase-3 (MMP-3) expression and syndecan 4 (SDC4) in intervertebral disc degeneration (IVDD) remains poorly defined. Wang et al (Am J Pathol 2014, 184:2560-2572) explored the regulatory relationship between cytokines, MMP-3, and SDC4 in rat and human nucleus pulposus cells. Tumor necrosis factor (TNF)- $\alpha$ and IL-1 $\beta$ cytokine treatment increased MMP- 3 expression and promoter activity. Loss-of-function studies confirmed that NF$\kappa \mathrm{B}, \mathrm{p} 38 \alpha / \beta 2 / \gamma / \delta$, and extracellular signal-regulated kinase (ERK)2, but not ERK1, contributed to cytokine-dependent induction of MMP3 promoter activity. Transforming growth factor- $\beta$ blocked TNF- $\alpha$-induced MMP-3 up-regulation and $S d c 4$ expression, counteracting the NF- $\kappa \mathrm{B}$ pathway. Controlling these pathways may slow the progression of IVDD and matrix catabolism. 